\title{
DECONSTRUYENDO LA UNIVERSALIDAD DE LA JUSTICIA SOCIAL: UNA PERSPECTIVA MULTICULTURAL HACIA NUEVOS HORIZONTES
}

\author{
Edil Torres Rivera ${ }^{1}$ \\ Wichita State University, Kansas, USA
}

Recientemente culminó el Congreso número 37 de la Sociedad Interamericana de Psicología, celebrado en la Habana, Cuba. El que se pudiera celebrar este Congreso en Cuba en tiempos de división socio-política e ideología fragmentada es por sí mismo un éxito y gran logro. También es significativo mencionar el discurso que pronunció el Coordinador General del Congreso el compañero Jorge Enrique Torralbas Oslé durante la Ceremonia de Clausura. En su discurso Jorge menciona el importante trabajo que tienen los psicólogos/as en tiempos de división e inestabilidad. Lo que me ha llevado a reflexionar acerca de en un punto en particular que fue mencionado por él y que fue eje central en la mayoría de los trabajos presentados durante el Congreso. Algo que también fue el tema central del discurso que pronuncié durante la celebración del 16 Congreso Caribeño de Psicología celebrado en Santo Domingo, República Dominicana. Este punto es el de justicia social. Durante este último año en mi posición como Editor de la Revista, me gustaría utilizar este espacio para tratar de deconstruir el término e invitar a los psicólogos/as interamericanos/as a mirar el concepto de justicia social más allá de las limitaciones impuestas al término por las razones que voy a exponer en siguientes páginas. También por primera vez solamente estoy escribiendo en español y no hare la traducción que usualmente hago en ingles.

La justicia social es un tema extensamente mencionado en la literatura psicológica norteamericana y existen, por lo menos, cinco manuales de justicia social en el mercado (por ejemplo, Jost \& Aaron, 2014; Toporek, Gerstein, Fouad, Roysircar, \& Israel, 2005). Para este editorial, he utilizando como base los artículos de Morrill (2018), Thrift y Sugarman (2018), y Raskin (2010) para desarrollar un argumento del por qué es necesario reconceptualizar el término justicia social.

El movimiento de justicia social en el campo de la psicología ha estado abriéndose paso durante los últimos 10 a 15 años; justicia social ha sido denominada la "quinta fuerza de la psicología" inmediatamente después de la consejería multicultural (D’Andrea \& Heckman, 2008; Ratts, 2012). También vemos este movimiento tomar fuerza en la División 17 de la Asociación Americana de Psicología (APA por sus siglas en inglés) con los trabajos de Vera y Speigh (2003) y Janet Helms (2003). También cabe señalar que de acuerdo con Thrift y Sugarman (2018) entre 2009 y 2015, PsyInfo revela que más de 3,000 publicaciones utilizaban la palabra clave justicia social en su base de datos. No obstante, incluso con esa popularidad y la mención constante del término, no se pueden encontrar en la literatura dos conceptos o definiciones similares de lo que es justicia social (Chung \& Bemak, 2011; Johnson \& Friedman, 2014; Morrill, 2018; Thrift \& Sugarman, 2018; Toporek, Gerstein, Fouad, Roysircar, \& Israel, 2005). Mis estudios en busca de poner en práctica la definición de psicología para la justicia social comenzó durante la revisión del trabajo de Strega y Brown en 2015 en la segunda edición de su libro "Investigación como resistencia: Revisando enfoques críticos, indígenas y anti-opresivos" (Strega \& Brown, 2015). El capítulo de Moosa-Mitha, "Situando teorías anti-opresivas con una perspectiva crítica y centrada en los diferentes puntos de vista” (Moosa-Mitha, 2015) me impresionó bastante ya que proporciona bases y conceptos para evaluar un verdadera teoría anti-opresiva. Sin embargo, es preciso señalar que las preguntas sobre qué es la justicia social en el campo de la psicología comenzaron mucho antes de mi búsqueda (Rectenwald, 2018). De hecho, al principio, e incluso en este

\footnotetext{
${ }^{1}$ Comentarios o preguntas acerca de este comentario editorial deben de ser dirigidos a Edil Torres Rivera. Email: etorresrivera@gmail.com
} 
documento, las palabras psicología y justicia social están separadas, debido a que Raskin (2010) indicó que existe una desconexión entre el proceso de psicología y el concepto de justicia social. Raskin (2010) indicó, además, que esta desconexión suponía que la justicia social es una construcción universal o teórica. Lo que significa que hablar de justicia social parece abarcar una serie de áreas que a veces parece que podrían aplicarse a todos, ¿Cuál es la pregunta? ¿Todos somos iguales? Si todos/as somos iguales, la necesidad de justicia social podría parecer como algo innecesario, porque la psicología consiste en ayudar a todas las personas necesitadas (Johnson \& Friedman, 2014). Una vez más, Raskin (2010) presentó un argumento sólido para considerar que la justicia social constituye una orientación teórica particular e introdujo el término psicología para la justicia social basado en cómo la opresión da forma a la experiencia humana. Por lo tanto, el objetivo del proceso de psicología para la justicia social consiste en redistribuir los recursos de una manera más equitativa, que es algo solamente materialista (Raskin, 2010).

Raskin (2010) ofreció algunas soluciones a las cinco críticas más comunes de la psicología para la justicia social después de proporcionar un marco teórico que fueron las siguientes: (a) la adopción del realismo ingenuo; (b) estar teóricamente no elaborado; (c) valores imponentes; (d) ser arrogante; (e) ir más allá de la gama de conveniencia de la psicología (consejería) (Raskin, 2010). Aunque todos los argumentos presentados por Raskin (2010) proponen soluciones al asunto de la psicología para la justicia social, el autor de este editorial no está de acuerdo con lo antes expuesto, ya que es importante examinar cada punto. En este particular, el trabajo de Thrift y Sugarman (2018) combinado con el de Raskin (2010) ofrecen argumentos para establecer una redefinición de la justicia social y la psicología que es importante y tiene dimensiones que podrían ayudar a una conceptualización más práctica y efectiva de la psicología para la justicia social.

El primer punto que Raskin (2010) presenta es que los psicólogos que practican la psicología para la justicia social a menudo son bastante ingenuos para el realismo. Es decir, Raskin ve a los psicólogos como personas que no son muy realistas y hasta pecan de esperar resultados que no son ni prácticos ni realistas. Adicionalmente también indica que muchos consejeros y psicólogos que usan un marco de justicia social usualmente solamente alientan a sus clientes a elevar su conciencia. Raskin (2010) ve esto como una imposición y un proceso paternalista. Sin embargo, es posible aprender de los movimientos de liberación, multiculturalidad y descolonización en los que las personas entienden que antes de poder hablar sobre liberación, aceptación y emancipación, deben pasar por un proceso de reflexión y liberación (Freire, 1996; Martin Baro, 2006; Montero, Sonn, \& Burton, 2017; Shin, 2015). El autor (Raskin) continúa y ofrece una alternativa que es muy similar a los enfoques de Foucault, Freire, y Martin Baró (Foucault, 2000; Freire, 1996; Martin Baro, 2006). No obstante, Raskin (2010) también continuó sugiriendo que la psicología para la justicia social solo contempla la opresión sin tener en cuenta que la psicología para la justicia social toma en consideración una serie de teorías cuando se trabaja con clientes y si bien la postura es anti-opresiva, no es el único que se trabaja o se considera (ver Potts \& Brown, 2015).

El segundo punto de Raskin (2010) sobre la crítica a la psicología para la justicia social, es que a la justicia social desde la perspectiva de la psicología aún le falta una teoría elaborada. De acuerdo con Raskin (2010) lo que necesita la psicología en términos de la justicia social es una teoría psicosocial de la que se deriven definiciones precisas y operativas sobre este concepto y sus implicaciones para la psicología. En este particular, podríamos sugerir que utilizar los puntos teóricos de la psicología de la liberación podría beneficiar a la psicología para la justicia social (ver Comas-Díaz \& Torres Rivera, in press).

El tercer punto de crítica de Raskin (2010) tiene que ver con la imposición de valores y sentido de estar de lado de la verdad y lo que es justo. Lo que le da al psicólogo/a que trabaja desde un enfoque de justicia social la idea de es aceptable imponer sus valores otros/as y que ellos/as saben más que otras personas. Es en este punto donde posiblemente las ideas de liberación entran a jugar un papel muy importante en los/as psicólogos/as que tienen como base la justicia social. Es decir, dentro del marco del modelo de Freire el proceso de reflexión es algo que debe de tomarse en cuenta y practicarse diariamente. Nadie posee la verdad universal por lo que la idea de imponerla suena como lo que Albert Memmi dijo en 
su famoso libro el "Retrato del Colonizado"(Memmi, 1957) - que lo mejor que puede hacer alguien que ha sido oprimido es oprimir. Por lo que repito, es imperante que los psicólogos/as que trabajan desde una perspectiva de justicia social utilicen el método Freireano de reflexión y problematizar (Montero, 2009).

El cuarto punto de crítica de Raskin (2010) es uno que me crea mucha disonancia pero que entra dentro en el contexto del nosotros/as contra ellos/as. Un sin número de psicólogos utiliza la justicia social como una bandera de arrogancia para sugerir que aquellos que usan un enfoque de justicia social son mejores que aquellos/as que no la practican. Es por esto que el movimiento de justicia social comenzó la idea del diálogo y el espacio para aquellos/as que no están de acuerdo. En este particular, deberíamos estar atentos a las voces que son diferentes, las que no se escuchan, y las que no tienen privilegio; el no hacerlo es pecar del mismo pecado del que acusamos a otros/as que no siguen el movimiento de justicia social.

La crítica final de Raskin (2010) tiene que ver con el alcance de la práctica de la consejería psicológica. Aunque hoy en día los psicólogos ya no se limitan a problemas del desarrollo personal y han entrado al ámbito clínico en términos de diagnóstico y tratamiento, para Raskin (2010) el moverse a los campos de la política y el activismo es sobre extenderse y sobre pasar los límites de la práctica. En este punto estoy en desacuerdo con Raskin (2010), ya que el mundo es político y política tiene que ver con entender el poder social y económico. Mas aún, política es entender que aquellos/as que contralan la economía controlan el poder y, por lo tanto, es imposible hacer un trabajo clínico efectivo sin entender esta dinámica de poder.

De Raskin (2010) pasamos a los argumentos esbozados por Erin Thrift y Jeff Sugarman (2018) con el fin de determinar si la justicia social es parte o no de la práctica de la psicología. El primer argumento de Thrift y Sugarmen (2018) es y cito "'La historia debe darle una pausa a los psicólogos que reclaman la justicia social como su misión" (p.15). "La justicia social se ha convertido en una "palabra clave cultural" y, a consecuencia, ya sea que los psicólogos se den cuenta o no, al invocar el término los impulsa a ellos y la disciplina (psicología) en un debate más amplio sobre la libertad humana, la responsabilidad individual y colectiva, y el papel del estado" (pp.15-16). Es decir, que, sin una definición concreta, los/as psicólogos/as y otras personas en el campo de la salud mental que utilizan el término justicia social entrarían en un proceso que puede ser muy simple y por lo tanto problemático.

Siguiendo la misma línea, Thrift y Sugarman (2018) explican que, aunque el significado del término "justicia social" ha cambiado a través de la historia en los países democráticos occidentales; el término se ha transformado a la par con los desarrollos culturales y acontecimientos que ha ganado mucha atención en los últimos años. Por lo tanto, la definición de justicia social carece de especificidad, claridad, y consenso. Sin embargo, el campo de la psicología se ha alineado con una misión de justicia social, dejando que algunos/as se pregunten qué significa exactamente esa misión.

Aunque muy pocas personas parecen conocer la historia multifacética del término justicia social en los países democráticos occidentales y de habla inglesa, Thrift y Sugarman (2018) presentan esta historia con algunas limitaciones (por ejemplo, no mencionar el papel de la teoría católica en el desarrollo de la justicia social y su definición), pero si proveen un análisis de su significado dentro del campo de la psicología (ver también a Rectenwald, 2018). Los autores, además, investigan el uso del término en los debates sobre la distribución económica justa de los bienes materiales y el poder y la reducción de los riesgos inherentes al sistema político/económico capitalista. El término justicia social no se usó originalmente en referencia a los movimientos por la igualdad de género o racial, ya que se dijo que esas injusticias "permanecieron invisibles" hasta alrededor de los años 70 (es importante mencionar que el término nunca fue utilizado por personalidades como Martin Luther King Jr. o Ignacio Martín Baró). La definición justicia social cambió durante los años 70, y comenzó a aplicarse a las desigualdades en bienes no materiales, como el reconocimiento de las diferencias y los problemas de identidad.

De acuerdo con Thrift y Sugarman (2018) el término justicia social se ha apropiado indebidamente con el aumento de la economía neoliberal en los años 70 y 80 . En este contexto, su significado se ha empleado para referirse a la justicia social como una virtud individual en lugar de un 
esfuerzo colectivo, un uso incompatible con el propósito original del término. Este cambio en el significado transforma las injusticias sociales en conflictos reconciliables por las prácticas privadas de caridad y el autocuidado en lugar de movimientos para la rendición de cuentas y la reforma de los sistemas y las corporaciones.

Sin embargo, a pesar de su origen, los diferentes puntos de vista y conceptualizaciones de la justicia social ahora representan la conexión de una lucha entre diferentes facciones políticas. Ahora, si no todas las opiniones y conceptualizaciones abordan suficientemente el término en su complejidad. Otra vez, Thrift y Sugarman (2018) argumentan que para que el término justicia social sea coherente con esta historia compleja, su utilización debe primero reconocer su desarrollo y significado históricos; y luego los desafíos contemporáneos que rodean su uso.

De acuerdo con Thrift y Sugarman (2018) la psicología, como campo, se ha alineado más estrechamente con algunas conceptualizaciones de la justicia social que otras. Dicho sea de paso, se ha criticado a los psicólogos/as por adoptar un enfoque de la justicia social basado en "políticas de identidad" en el que se abordan de manera reduccionista los problemas de identidad y reconocimiento, mientras que pasan por alto la intersección de identidad con desigualdades económicas y preocupaciones estructurales más amplias. No es sólo que el privilegio de los puntos de vista de la justicia social centrados en la identidad oscurezca las desigualdades económicas asociadas con el capitalismo, sino que adopta una postura cómplice que permite la perpetuación de estas injusticias. En este sentido, los/as psicólogos/as se han alineado predominantemente con un enfoque reduccionista de la justicia social y al hacerlo, han debilitado su misión declarada de justicia social.

Al reclamar pericia en la definición y tratamiento de problemas psicológicos, el campo de la psicología tiene una influencia considerable en el uso y la comprensión de la justicia social. Como resultado, la confusión sobre el significado de la justicia social tiene implicaciones para los/as psicólogos/as interesados/as en lograr este objetivo, pero también tiene consecuencias políticas, sociales y económicas mucho más amplias. Cuando los/as psicólogos/as promueven la idea de que el sufrimiento psicológico es un estado que puede resolverse exclusivamente mediante intervenciones individuales, como la psicoterapia, los cambios de comportamiento o los tratamientos con medicamentos, los problemas estructurales pueden ignorarse y perpetuarse.

Thrift y Sugarman (2018) continúan describiendo cómo las explicaciones psicológicas específicas han desviado a los individuos de la participación política y han evitado reformas sistémicas. Por ejemplo, el sufrimiento y la subyugación de las mujeres se explica por la histeria; la discriminación racial se justifica por la inteligencia inferior de las personas de color; la homosexualidad se clasifica como un trastorno mental en el DSM; las familias no occidentales se describen como desordenadas; y el impacto negativo de la pobreza en el rendimiento académico de la niñez se ha considerado como falta de autodisciplina o como déficit en otras características internas. En este particular, los problemas estructurales no tan solo se ocultan en estas explicaciones, sino que se reemplazan con interpretaciones que asignan responsabilidad exclusiva a individuos o características individuales. Thrift y Sugarman (2018) también señalan que los psicólogos se benefician de una promoción individualizada de la justicia social; y que la psicología como campo está incrustada en la economía de mercado por lo que los problemas que surgen son problemas del individuo y así pueden aumentar la demanda de servicios psicológicos. Como consecuencia, puede haber un pequeño incentivo profesional o económico para que los/as psicólogos/as conceptualicen las dificultades personales que no sean los individuos, debido a que existe una ganancia material en continuar la tradición de que todos los problemas de las personas son el producto de las propias personas falta de organización y habilidad baja de lidiar con los problemas diarios.

Finalmente, Thrift y Sugarman (2018) ofrecen algunas recomendaciones que están a la par con mis estudios y creencias en cómo la justicia social desempeña un papel importante en el campo de la psicología en general, pero mucho más crítico en lo clínico. En este particular, los autores promueven el modelo de Fraser (2009). Fraser (2009) defiende un principio de paridad participativa, lo que significa que todas las injusticias deben considerarse como violaciones de la justicia social. La justicia social y las violaciones a la justicia social se evalúan dentro de este marco en términos de su efecto en la capacidad de 
una persona para participar social y políticamente en igualdad de condiciones con sus compañeros (Fraser, 2009). Para abordar los problemas contemporáneos que rodean las demandas de justicia social, la globalización de justicia social debe estar enmarcadas más allá del interés de una nación-estado y deben ser capaces de reconocer las injusticias globales cometidas por las empresas transnacionales.

Para el campo de la psicología pueda aplicar la justicia social en términos del principio de paridad participativa, Thrift y Sugarmen (2018) sugieren una reflexión sobre la siguiente pregunta: "¿Cómo la teorización psicológica, la investigación o las intervenciones ayudan a crear acuerdos sociales, culturales, políticos y económicos que permiten a las personas participar en un nivel igual con sus compañeros?" (p.17). De acuerdo con los autores, la respuesta del campo de la psicología debe ir más allá de simplemente promover y aumentar el acceso a los servicios psicológicos; la justicia social exige una reforma a gran escala de los servicios psicológicos para abordar los problemas sociopolíticos y económicos, en lugar de socavarlos.

\section{Conclusiones}

En conclusión, los trabajos de Thrift y Sugarman (2018), al igual que los de Raskin (2010), y Moosa-Mitha (2015) deberían combinarse. La combinación de estas ideas no solamente produciría una definición más adecuada de lo que la psicología para la justicia social seria, sino que además estaría añadiendo componentes de métodos anti-opresivos y liberatorios, por encima de lo podríamos utilizar de la psicología de la liberación. Esta acción nos daría una ontología de la opresión que nos permite entender y reconocer la experiencia de opresión individual, sin perder el sentido de las experiencias colectivas. En otras palabras, la experiencia individual no es ni mayor ni menor que la colectiva. Durante una conversación con una joven en la Republica Dominicana (Leticia) esta me comentó que la gente es complicada. Esto me recordó que todos/as reconocemos la complejidad humana, pero por una razón u otra parece que tenemos una tendencia a simplificarla. Es por eso que al movernos hacia un entendimiento y desarrollo de una teoría aplicada de justicia social debemos también definir la ontología de la justicia social como algo que es múltiple, fluida, e interseccional.

Igualmente, la epistemología de la justicia social debe de basarse en que el conocimiento debe de ser comprendido como subjetivo y basado en las experiencias de las personas. Al mismo tiempo, el conocimiento es subyugado y situacional. Es decir, situado en la localización social de la persona como resultado de los privilegios y la opresión que se ha experimentado. Igualmente es importante reconocer que el conocimiento es solamente parcial y que no es posible atener todo el conocimiento.

Finalmente quiero recalcar el último argumento señalado por Thrift y Sugarman (2018) en el que se enfatiza que la justicia social exige una reforma a gran escala de los servicios psicológicos para poder abordar los problemas sociopolíticos y económicos en lugar de socavarlos. Con el entendimiento de que existen cosas que son complicadas, fluidas, parciales, y múltiples. Tenemos que entender que abrazar la condición humana es también moverse de nuestra zona de comodidad. Esperemos que el/ psicólogo interamericano pueda ver nuevos horizontes que no tienen que ser marcados por reglas que no representan las personas que servimos, ayudamos, y representamos. 


\section{References}

Chung, R. C.-Y., \& Bemak, F. (2011). Social Justice Counseling: The Next Steps Beyond Multiculturalism. Thousand Oaks: CA: Sage Publications.

Comas-Diaz, L., \& Torres Rivera, E. (n.d.). Liberation Psychology: Theory, Method, Practice, and Social Justice. (L. Comas-Díaz \& E. Torres Rivera, Eds.). Washington: DC: America Psychologicla Association.

D’Andrea, M., \& Heckman, E. F. (2008). Contributing to the Ongoing Evolution of the Multicultural Counseling Movement: An Introduction to the Special Issue. Journal of Counseling \& Development, 86(3), 259-260. https://doi.org/10.1002/j.1556-6678.2008.tb00507.x

Foucault, M. (2000). Power: Essential Works of Foucault, 1954-1984. New York: New Press.

Fraser, N. (2009). Scales of justice: Reimagining political space in a globalizing world. New York: Columbian University Press.

Freire, P. (1996). Pedagogy of the oppressed (Rev. ed.). New York: NY: Penguin Group.

Helms, J. E. (2003). A Pragmatic View of Social Justice. The Counseling Psychologist, 31(3), 305-313. https://doi.org/10.1177/0011000003031003006

Johnson, C. V., \& Friedman, H. L. (2014). Set Introduction. In J. Diaz, Z. Franco, \& K. Nastasi, Bonnie (Eds.), The Handbook of Social Justice and Psychology (Volume 1:, pp. xv-xx). Santa Barbara: Praeger.

Jost, J. T. \& Kay, A. C. (2014). The history of psychology and social jusitce. In D. Jeannette, Z. Franco, \& B. K. Nastasi (Eds.), The Palgrave Handbook of Critical Social Psychology: Fundamental issues and special populations (Volumen 1, pp. 3-32). Santa Barbara: Praeger ABC-CLIO, LLC.

Martin Baro, I. (2006). Hacia una psicologia de liberacion. Revista Electrónica de Intervención Psicosocial y Psicología Comunitaria, 1(2), 7-14.

Memmi, A. (1957). The colonizer and the colonized. Trans. Howard Greenfeld. Boston: Beacon, (1), 86. https://doi.org/10.4324/9781315065670

Montero, M. (2009). Methods for liberation: Critical consciousness in action. In M. Montero \& C. Sonn, Christopher (Eds.), Psychology of liberation: Theory and applications (pp. 73-91). New York: NY: Springer.

Montero, M., Sonn, C. C., \& Burton, M. (2017). Community psychology and liberation psychology: A creative synergy for an ethical and transformative praxis. In M. A. Bond, I. Serrano-García, C. B. Keys, \& M. Shinn (Eds.), APA handbook of community psychology: Theoretical foundations, core concepts, and emerging challenges, Vol. 1 (pp. 149-167). Washington, DC, US: American Psychological Association. https://doi.org/10.1037/14953-007

Moosa-Mitha, M. (2015). Situating anti-oppression theories within critical and difference-centred perspective. In S. Strega \& L. Brown (Eds.), Research as resistance: Revisting critical, indigenous, and anti-oppresive approaches (2nd ed, pp. 65-96). Toronto: Canadian Scholars' Press Women’s Press.

Morrill, Z. (2018). What does social justice really mean for psychologies. Retrieved September 24, 2018, from https://www.madinamerica.com/2018/09/social-justice-really-mean-psychologists/

Potts, K., \& Brown, L. (2015). Becoming an Anti-Oppressive Researcher. In S. Strega \& E. Brown (Eds.), Research as resistance : critical, indigenous and anti-oppressive approaches (2nd Ed, pp. 17-41). Toronto: Ontario: Canadian Scholars' Press Women's Press.

Raskin, J. D. (2010). Constructing and deconstructing social justice counseling. In Jonarthan D. Raskin, S. K. Bridges, \& R. A. Neimeyer (Eds.), Studies in meaning 4: Constructivist perspectives on theory, practice, and social jusitce (pp. 247-276). New York: Pace University Press.

Ratts, M. J. (2012). 5 forces of counseling and psychotherapy, 5, fifth force: The social jusitce counseling session. Micortraining Associates.

Rectenwald, M. (2018, August). On the origins and character of "social justice." New English Review, 28.

Shin, R. Q. (2015). The Application of Critical Consciousness and Intersectionality as Tools for 
Decolonizing Racial/Ethnic Identity Development Models in the Fields of Counseling and Psychology BT - Decolonizing “Multicultural” Counseling through Social Justice. In R. D. Goodman \& P. C. Gorski (Eds.) (pp. 11-22). New York, NY: Springer New York. https://doi.org/10.1007/978-1-4939-1283-4_2

Strega, S., \& Brown, L. (2015). Research as resistance : revisiting critical, indigenous, and antioppressive approaches. (S. Strega \& L. Brown, Eds.) (2nd Ed.). Toronto: Canadian Scholars' Press Women's Press. Retrieved from https://womenspress.cspi.org/

Thrift, E., \& Sugarman, J. (2018). What is social justice? Implications for psychology. Journal of Theoretical and Philosophical Psychology, 39(1), 1-17. https://doi.org/10.1037/teo0000097

Toporek, R. L., Gerstein, L., Fouad, N., Roysircar, G., \& Israel, T. (2005). Handbook for social justice in counseling psychology: Leadership, vision, and action. Sage Publications.

Vera, E. M., \& Speight, S. L. (2003). The Counseling Psychologist Expanding Our Roles, 31(3), 253272. https://doi.org/10.1177/0011000002250634 